\title{
A SUBCHANNEL COARSENING METHOD FOR SERPENT2-SUBCHANFLOW APPLIED TO A FULL-CORE VVER PROBLEM
}

\author{
Manuel García ${ }^{1}$, Diego Ferraro ${ }^{1}$, Ville Valtavirta ${ }^{2}$, Riku Tuominen $^{2}$, Uwe Imke $^{1}$, \\ Luigi Mercatali $^{1}$, Victor Sanchez-Espinoza ${ }^{1}$, Jaakko Leppänen ${ }^{2}$ \\ ${ }^{1}$ Karlsruhe Institute of Technology (KIT) \\ Hermann-von-Helmholtz-Platz 1, 76344 Eggenstein-Leopoldshafen, Germany \\ ${ }^{2}$ VTT Technical Research Centre of Finland Ltd. \\ Tietotie 3, Espoo, FI-02044 VTT, Finland \\ manuel.garcia@kit.edu,diego.ferraro@kit.edu
}

\begin{abstract}
This work presents a methodology to coarsen subchannel models to accelerate largescale Serpent2-SUBCHANFLOW calculations. The method is based on first building a fully detailed subchannel model and then combining subchannels into larger channels with condensed hydraulic parameters. To quantify the accuracy and performance of this scheme, a full-core VVER-1000 problem is simulated with Serpent2-SUBCHANFLOW using two types of coarsened models, as well as a standard subchannel model as reference.
\end{abstract}

KEYWORDS: Serpent2, SUBCHANFLOW, Multiphysics, Subchannel coarsening, VVER.

\section{INTRODUCTION}

As part of a global trend to develop high-fidelity applications for reactor physics simulations, the EU Horizon $2020 \mathrm{McSAFE}$ project [1] was set to develop multiphysics tools based on the Monte Carlo particle transport method, with the objective of tackling large-scale pin-by-pin depletion and transient problems.

In this framework, a coupling scheme for Serpent2 [2], a continuous-energy Monte Carlo code, and SUBCHANFLOW (SCF) [3], a subchannel thermalhydraulics code, has been developed with the final aim at performing full-core high-fidelity depletion calculations for Light Water Reactors (LWR) [4]. The main features of this coupled system are described in Section 2.

The default modelling approach in the McSAFE project is a fully pin-by-pin and subchannellevel representation, which leads to massive memory and performance requirements for the target problems. It is therefore desirable to have as an alternative a simplified scheme which provides a faster solution with moderate impact on the results of interest, namely pin-level safety parameters such as Departure from Nucleate Boiling Ratio (DNBR) and peak fuel and cladding temperatures, and pin-by-pin depletion data. This is particularly important for SCF, given that its flow solver is 
not parallelized, whereas Serpent 2 features hybrid MPI-OpenMP parallelization, allowing its use of High Performance Computing (HPC) resources.

In this work, a subchannel coarsening method for SCF is proposed, with the methodology presented in Section 3. This scheme is applied to a steady-state calculation of a full-core VVER problem, described in Section 4. The results to evaluate the accuracy of the coarsening method are shown in Section 5, along with runtime data to quantify the gains in terms of performance.

\section{SERPENT2-SUBCHANFLOW}

The Serpent2-SCF calculation scheme is based on the standard neutronic-thermalhydraulic iterative method. The power P calculated by Serpent 2 is used to perform the thermalhydraulic calculation in SCF, which in turns provides the coolant density $\rho_{\text {cool }}$ and temperature $T_{\text {cool }}$, as well as the fuel temperature $T_{\text {fuel }}$, as feedback for the neutronics. Successive Serpent 2 and SCF calculations are performed until a convergence criterion is reached, in this case using $\mathrm{P}, \rho_{\text {cool }}$ and $\mathrm{T}_{\text {fuel }}$, as well as the multiplication factor $\mathrm{k}_{\mathrm{eff}}$, as convergence variables. To improve the stability of the coupled solution the Stochastic Approximation method, a type of relaxation, is used.

The exchange of feedback variables is done using fields defined on unstructured meshes superimposed to the geometry, as shown in Figure 1 for a SCF model of a VVER-1000 fuel assembly, where the subchannel model has two superimposed meshes, one for the coolant and one for the fuel. The meshes and fields correspond to the MEDCoupling library [5], which also provides advanced interpolation methods which are used to map the Serpent 2 and SCF geometries.

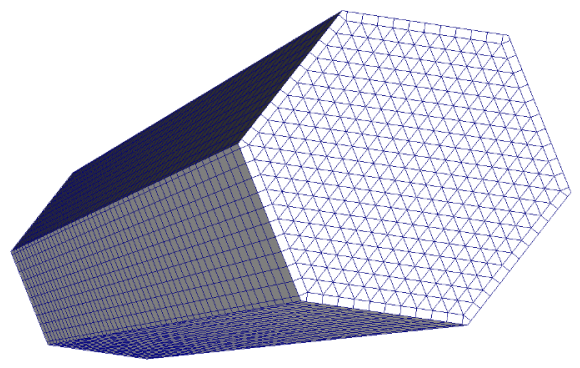

(a) SCF coolant mesh.

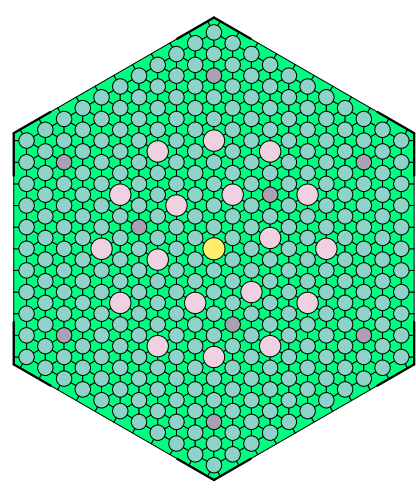

(b) SCF model.

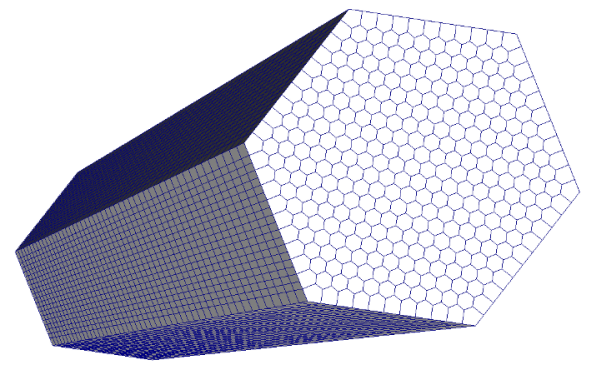

(c) SCF fuel mesh.

Figure 1: SCF model and meshes for a VVER-1000 fuel assembly.

\section{SUBCHANNEL COARSENING METHOD}

Given that the most time-consuming part of a SCF calculation, especially for large problems, is the pressure-velocity solution, the decision was made to only coarsen the coolant domain, i. e. the subchannel model. A pin-by-pin description of the fuel rods is maintained to obtain a detailed solution for the safety parameters and the Doppler feedback for Serpent2. 
The subchannel coarsening method for SCF is performed as a preprocessing step, i. e. before the calculation and implemented outside the SCF source code, and can be summarized as:

1. Build the subchannel model and calculate the hydraulic parameters and flow connectivity.

2. Superimpose a polar mesh defining the coarse zones.

3. Combine all subchannels in each coarse zone to build an equivalent channel, condensing the hydraulic and connectivity data.

As an example, Figure 2 shows the original subchannel model (2a) and the resulting coarsening (2b) for a subsection of the VVER-1000 full-core model described in Section 4. In this case the fine model is maintained in a selected region, while the rest is coarsened.

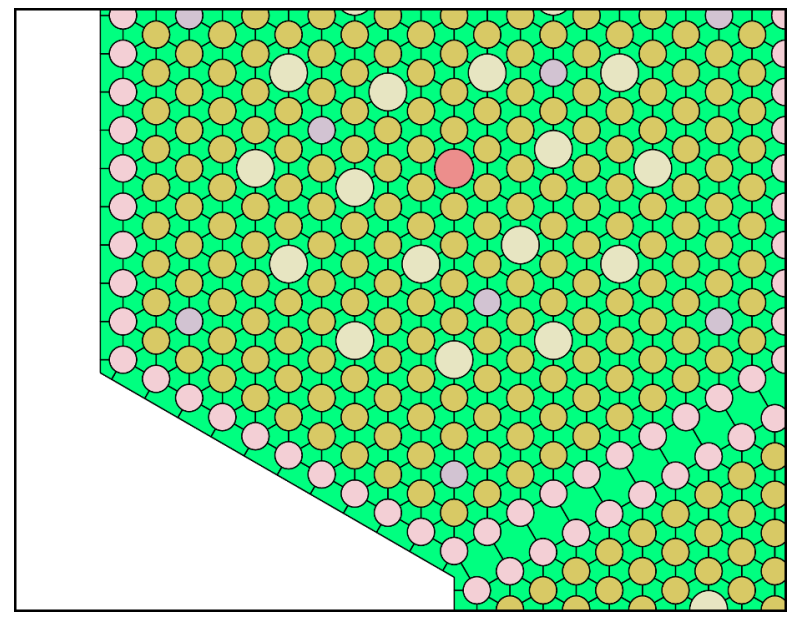

(a) Subchannel model.

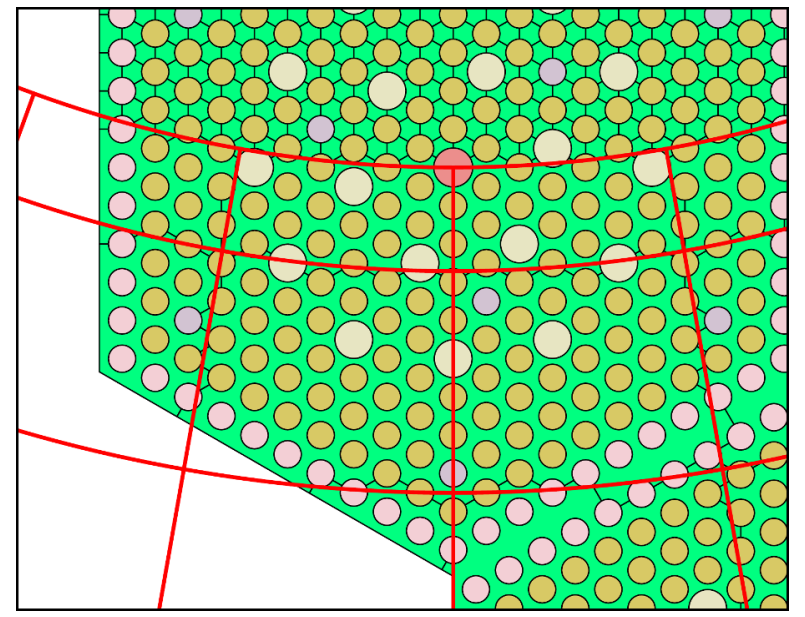

(b) Coarsened model.

Figure 2: Subchannel coarsening method.

The hydraulic parameters, i. e. flow area, and wetted and heated perimeter, of the coarse channels can be calculated simply adding these quantities for the original subchannels. Condensing the connectivity data, which is used to calculate the cross flow between channels, is not as straight forward, and is done taking into account only the original subchannels that lay directly in the boundary between each pair of coarse channels. The gap length, i. e. the free flow area from one channel to a neighbor, is calculated as a sum over the boundary subchannels, resulting in the physical gap between rods. The distance associated with a connection is taken as the average distance between the boundary subchannels. Hence, each cross-flow connection is characterized by local subchannel data in the vicinity of the gap, and the empirical values for the turbulent mixing and cross-flow resistance coefficients, valid for standard subchannel models, are still used.

\section{TEST PROBLEM AND MODELLING APPROACH}

The test case selected is a full-core VVER-1000 benchmark based on the second unit of the Khmelnitsky nuclear power plant [6]. The core consists of 163 TVSA fuel assemblies of five different 
types (390GO, 39AWU, 22AU, 30AV5 and 13AU), with different enrichments and burnable poisons. The operating conditions correspond to the Hot Full Power state at 3000MWth, without xenon. A complete description of the problem can be found in the benchmark specification [6].

The Serpent 2 geometry consists of the full core with a pin-by-pin description and a radial reflector, as shown in Figure $3 \mathrm{a}$ for a quarter of the model. Axially, the active length is composed of sections with and without grid spacers, and axial reflectors are included on the top and bottom of the core (Figure 3b). For each transport calculation 2000 active cycles of $10^{6}$ neutrons are used, with the source calculated initially with 300 inactive cycles and corrected before each iteration with 100 additional cycles. The power is tallied using a pin-by-pin mesh with 30 axial nodes.

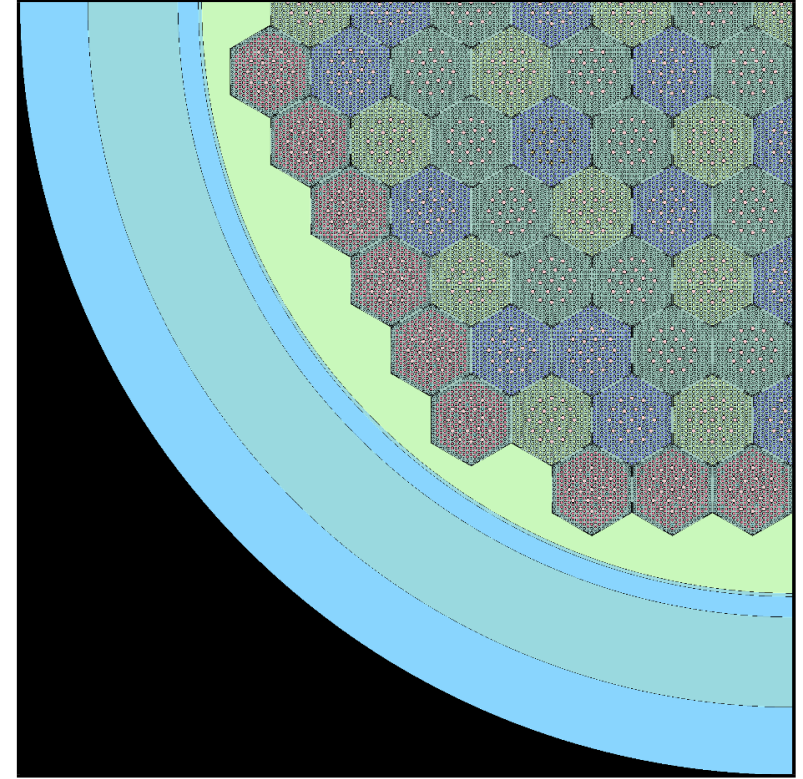

(a) Radial cut (one quarter).

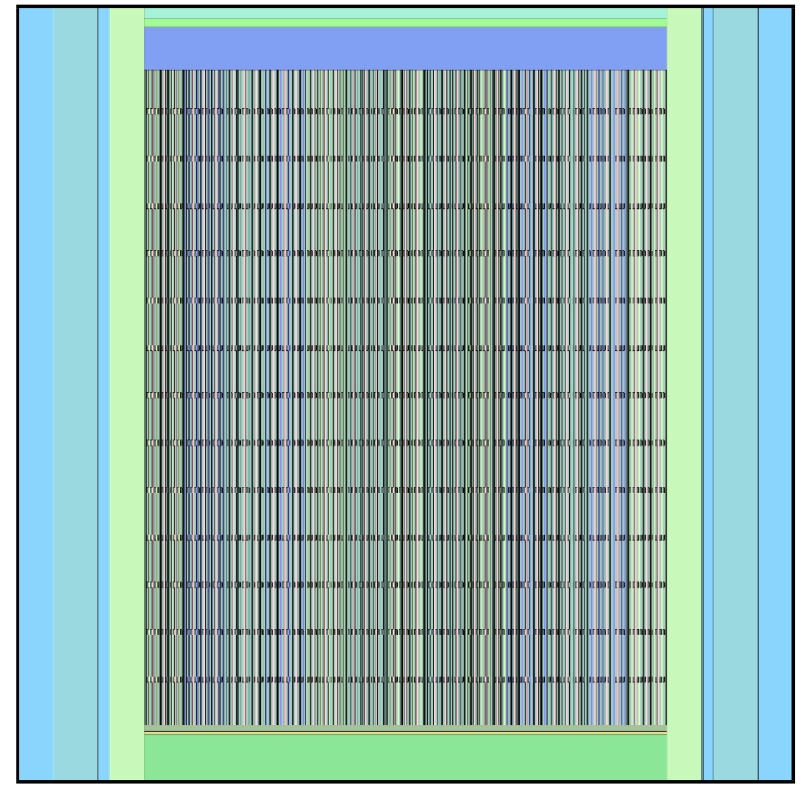

(b) Axial cut.

Figure 3: Serpent2 model.

To study the proposed methodology, a standard (fine) coolant-centered subchannel model is used in SCF as reference solution and two types of coarsening schemes are considered. The first (mixed) model features subchannel resolution in the region with the highest power and lowest DNBR, located in the corners of the core, with the coarsening method applied gradually outwards from this area, as shown in Figure 4a. Since the core has a 1/6 symmetry around the centroid, having a fine model in one of the corner suffices to calculate local safety parameters at subchannel level. The second model, shown in Figure $4 \mathrm{~b}$ is fully coarsened, without subchannel resolution anywhere. In all models the active length is discretized in 30 axial levels.

\section{RESULTS AND DISCUSSION}

Figure 5 shows the coolant temperature and the power calculated using the fine subchannel model in SCF. This solution is taken as reference to evaluate the accuracy of the coarsened models. 


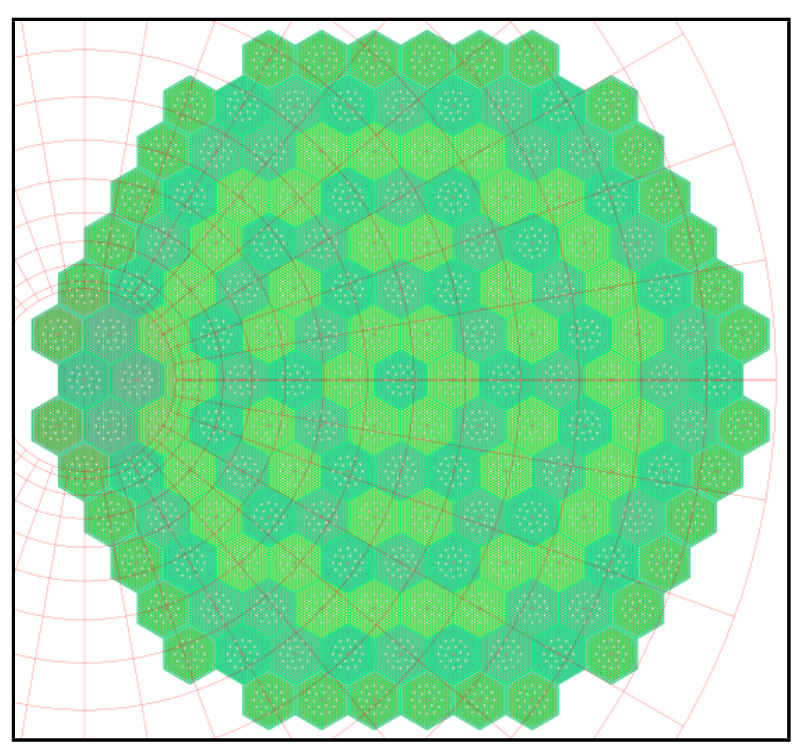

(a) Mixed model.

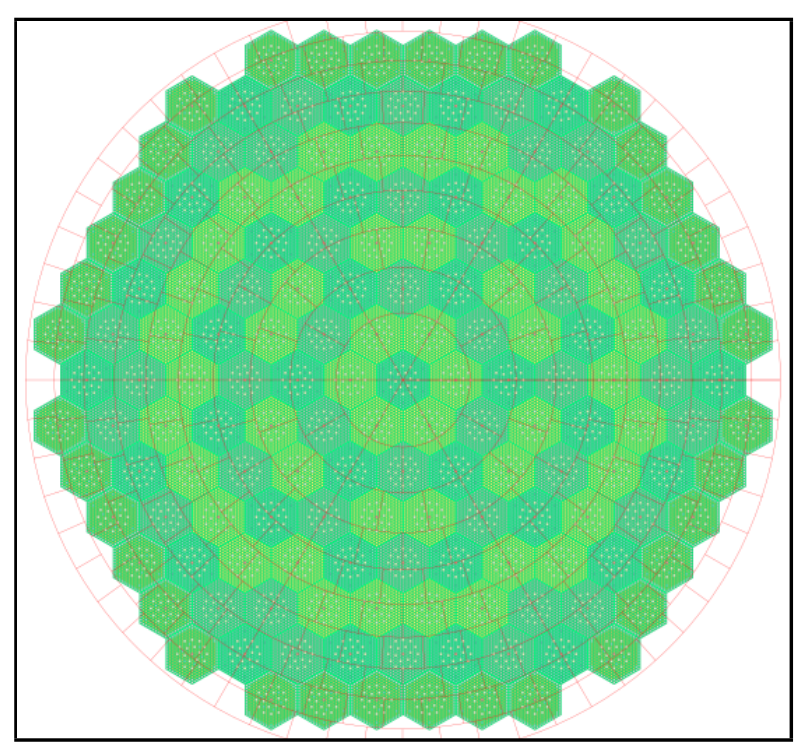

(b) Coarse model.

Figure 4: SUBCHANFLOW coarsened models.

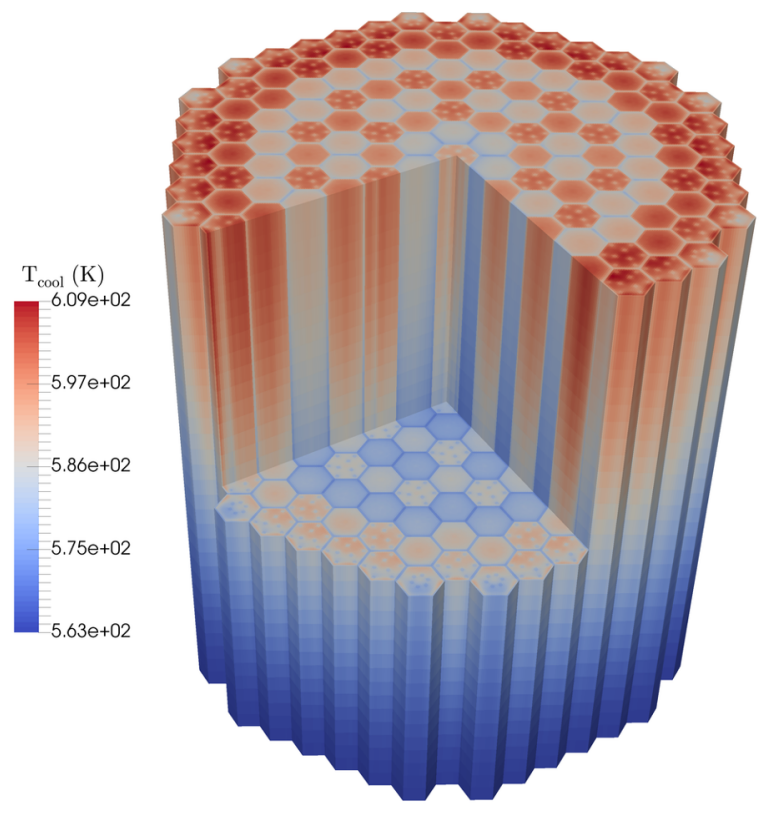

(a) Coolant temperature.

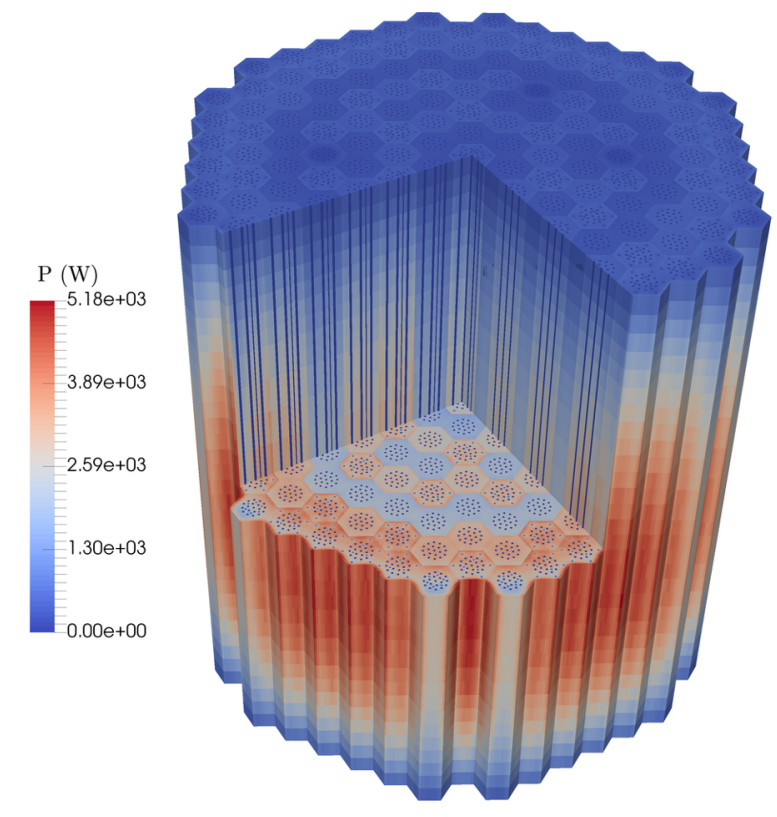

(b) Power.

Figure 5: Results using the reference SCF model.

Table 5 summarizes the main global results for the three models, as well as the differences for the mixed and coarse SCF models with respect to the reference solution. For the mixed model the safety parameters are taken from the zone with subchannel resolution. Overall the results are in 
very good agreement and within the typical uncertainties associated with safety parameters.

\begin{tabular}{|c|c|c|c|}
\hline Model & Fine & Mixed & Coarse \\
\hline $\mathrm{k}_{\text {eff }}$ & $1.02580 \pm 2 \mathrm{pcm}$ & $1.02615 \pm 2 \mathrm{pcm}(35 \mathrm{pcm})$ & $1.02611 \pm 2 \mathrm{pcm}(31 \mathrm{pcm})$ \\
DNBR & 2.59 & $2.68(3.2 \%)$ & $2.64(1.6 \%)$ \\
Power peak factor & 2.80 & $2.77(-0.8 \%)$ & $2.79(-0.3 \%)$ \\
Peak $\mathrm{T}_{\text {fuenterline }}^{\text {cente }}$ & $1830.0 \mathrm{~K}$ & $1823.8 \mathrm{~K}(-6.2 \mathrm{~K})$ & $1826.4 \mathrm{~K}(-3.6 \mathrm{~K})$ \\
Peak $\mathrm{T}_{\text {clad }}$ & $650.2 \mathrm{~K}$ & $649.7 \mathrm{~K}(-0.5 \mathrm{~K})$ & $647.9 \mathrm{~K}(-2.4 \mathrm{~K})$ \\
\hline
\end{tabular}

Table 1: Global results and differences with respect to the reference model.

Figure 6 shows the local differences in the coolant temperature averaged axially for the two coarsened models with respect to the fine model, which in both cases are below 10K. These local errors are of course expected, since in the coarse channels the subchannel resolution is lost. For the mixed model, the solution essentially matches the reference one in the region with subchannel detail.

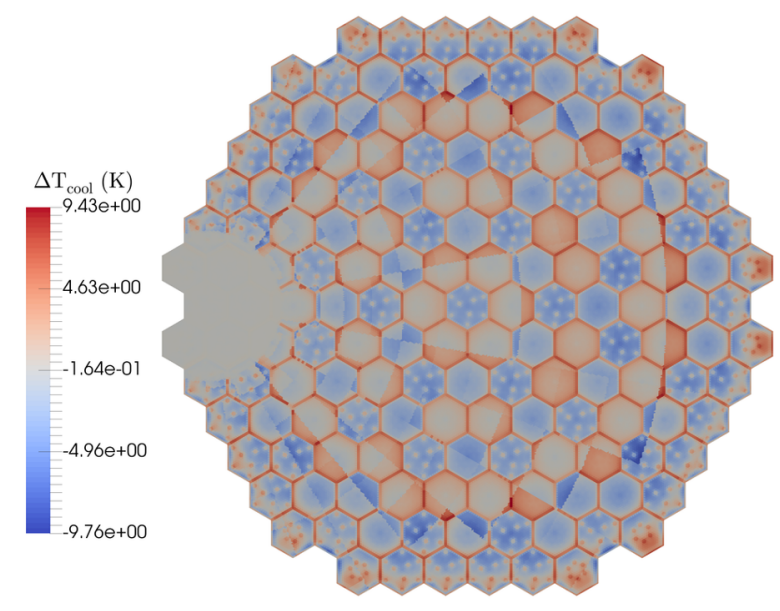

(a) Mixed model.

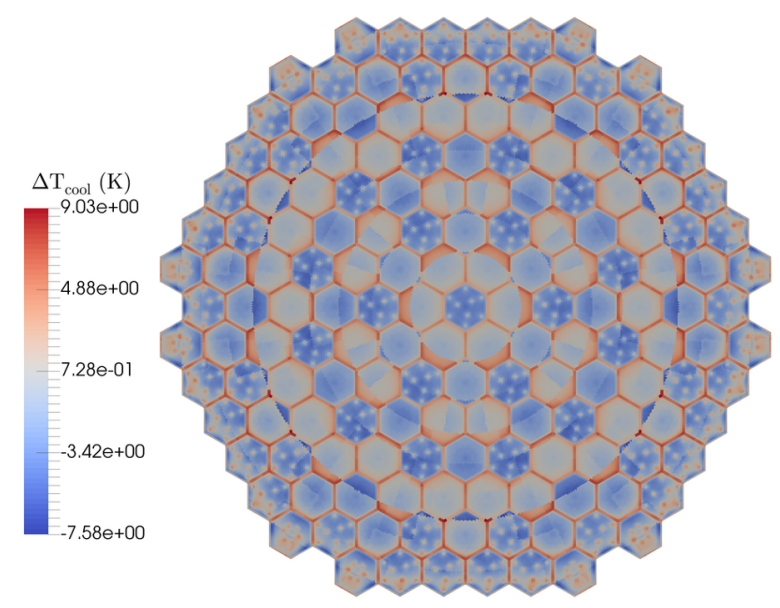

(b) Coarse model.

Figure 6: Differences in the coolant temperature with respect to the fine model.

The differences in the pin power distribution (Figure 7) are lower than $4 \%$ everywhere, and can be attributed to two factors. Firstly, there seems to be a small tilt in the overall profile towards the right in both cases, likely due to the statistical uncertainty of the Monte Carlo solution and not to the thermalhydraulic solution, since the coarse model in particular has 1/6 symmetry. Secondly, the power tends to shift locally following the coolant temperature, as can be observed analyzing figures 6 and 7.

Figure 8 shows the differences in fuel temperature distribution, which are lower than $10 \mathrm{~K}$ for all pins and are consistent with the deviations in the coolant temperature and power. 


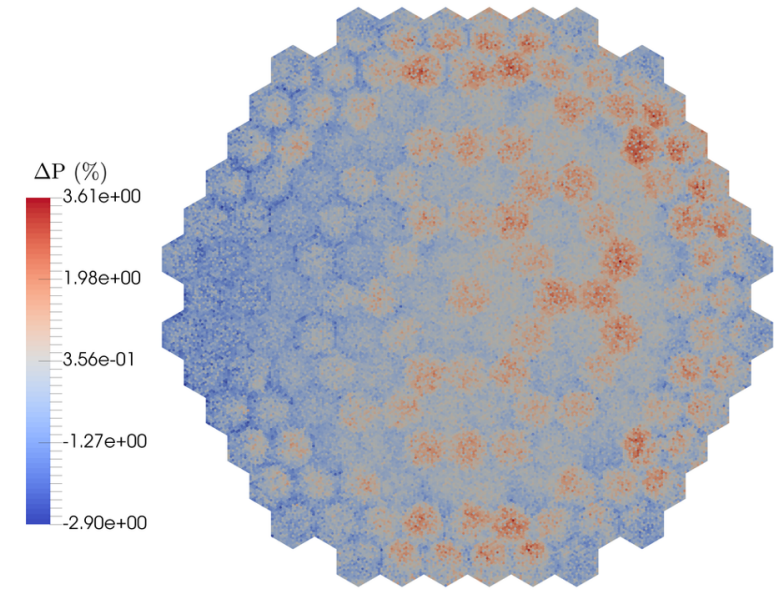

(a) Mixed model.

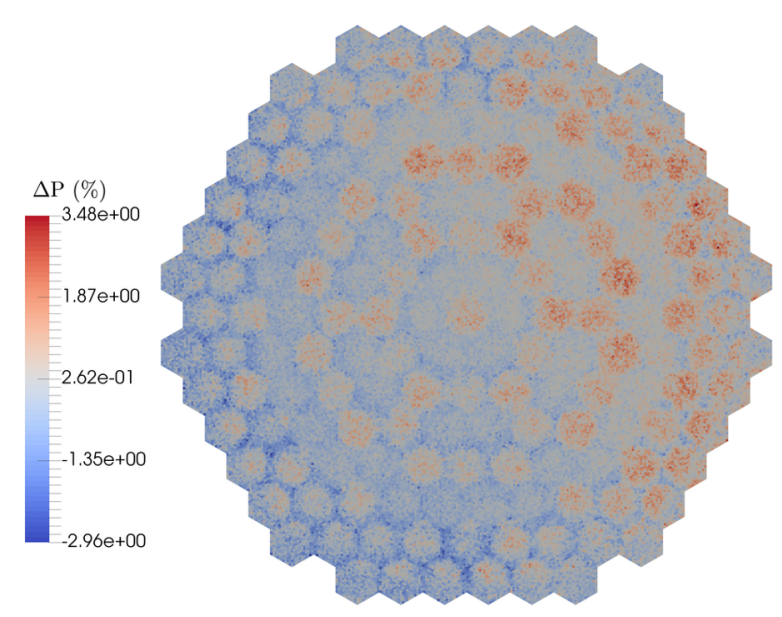

(b) Coarse model.

Figure 7: Differences in the power with respect to the fine model.

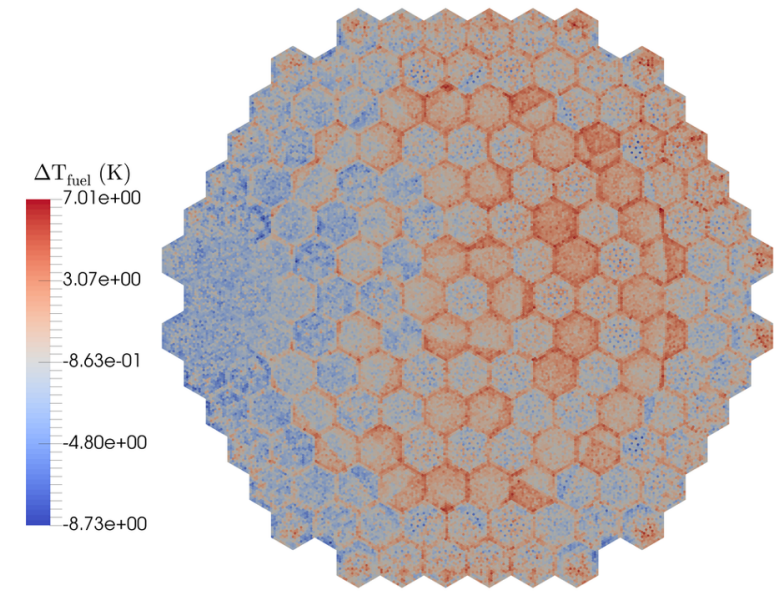

(a) Mixed model.

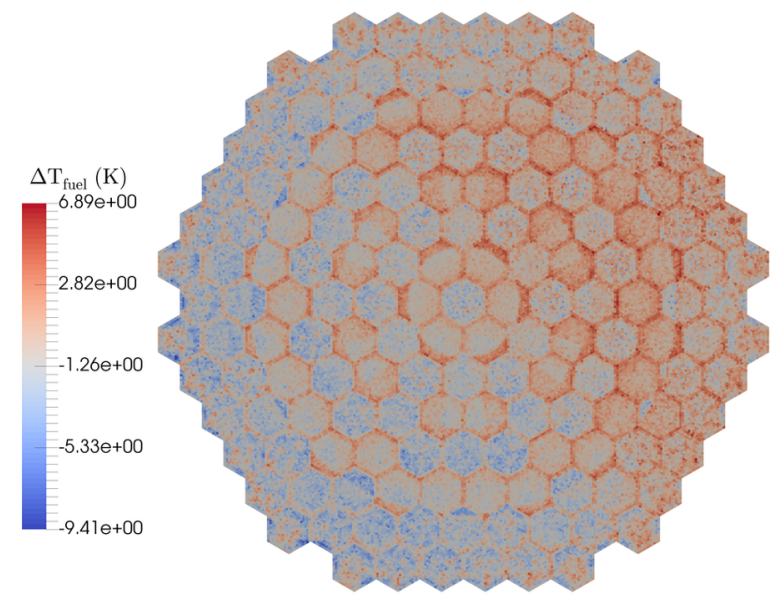

(b) Coarse model.

Figure 8: Differences in the fuel temperature with respect to the fine model.

\subsection{Performance}

Table 2 shows the main performance data for the three models considered. The calculation time per SCF iteration is reduced by $74.9 \%$ and $81.5 \%$ using the mixed and coarse models respectively. This represents a significant speedup in the coolant calculation, considering that in the three models all the rods are simulated, which represents a fixed computational cost independent of the pressure-velocity solver. Due to this reduction in the SCF calculation time, the fraction of the total runtime spent in Serpent 2 increases, which in turn is expected to improve the parallel efficiency of the overall system. These results were obtained in a single computational node with 48 Intel(R) Xeon(R) Gold 5118 CPUs, with Serpent 2 running on 48 OpenMP threads and SCF sequentially. 


\begin{tabular}{|c|c|c|c|}
\hline Model & Fine & Mixed & Coarse \\
\hline Time per SCF iteration & $89.4 \mathrm{~min}$ & $22.5 \mathrm{~min}(-74.9 \%)$ & $16.6 \mathrm{~min}(-81.5 \%)$ \\
SCF fraction & $10.8 \%$ & $2.9 \%$ & $2.2 \%$ \\
Serpent2 fraction & $87.8 \%$ & $88.4 \%$ & $91.0 \%$ \\
\hline
\end{tabular}

Table 2: SCF iteration time and fraction of the total runtime spent in each code.

\section{CONCLUSIONS}

A subchannel coarsening methodology for SCF has been formulated to accelerate Serpent2-SCF full-core simulations for LWRs. The scheme is based on building a standard subchannel model and combining subchannels according to a superimposed mesh. In this way, the hydraulic data can be calculated for the fine model and later condensed for the coarse channels.

This method was applied to a full-core VVER-1000 problem using two types of coarsened models, one combining subchannel resolution and coarse channels and one fully coarse, and a standard subchannel model as reference. The results for both simplified models are in very good agreement with the fine model, with minor differences in all the global parameters and deviations of less than $4 \%$ in the pin power distribution.

\section{ACKNOWLEDGMENTS}

This work was done within the McSAFE project which is receiving funding from the Euratom research and training programme 2014-2018 under grant agreement No 755097.

\section{REFERENCES}

[1] L. Mercatali, et al. "The EC McSAFE Project: High Performance Monte Carlo Methods for Safety Demonstration - Status and Perspectives." International Multi-Physics Validation Workshop, North Carolina State University, Raleigh, USA, June 14-15, 2018.

[2] J. Leppänen, et al. "The Serpent Monte Carlo code: Status, development and applications in 2013." Annals of Nuclear Energy, volume 82, pp. 142-150 (2015).

[3] U. Imke, et al. "Validation of the Subchannel Code SUBCHANFLOW Using the NUPEC PWR Tests (PSBT)." Science and Technology of Nuclear Instalations, volume 2012 (2012).

[4] M. García, et al. "Development of an Object-oriented Serpent2-SUBCHANFLOW Coupling and Verification with Problem 6 of the VERA Core Physics Benchmark." International Conference on Mathematics and Computational Methods applied to Nuclear Science and Engineering (M\&C 2019), Portland, Oregon, USA, August 25-29, 2019 (2019).

[5] CEA/DEN, EDF R\&D, and OPEN CASCADE. "SALOME Platform Documentation: MEDCoupling User's Guide.” https://docs.salome-platform.org/7/dev/MEDCoupling/index.html.

[6] T. Lötsch, et al. "Proposal of a Benchmark on core burnup follow calculations for a VVER1000 reactor core." Proceedings of the 19th Symposium of AER on VVER Reactor Physics and Reactor Safety, Varna, Bulgaria (2009). 